\title{
Heterogeneous Cell Types in Single-cell-derived Clones of MCF7 and MDA-MB-231 Cells
}

\author{
GUL NABI KHAN, EUN JIN KIM, TAE SEOP SHIN and SANG HO LEE \\ Division of Life Sciences, Korea University, Seoul, Republic of Korea
}

\begin{abstract}
Background/Aim: Variations in cell phenotype in a single-cell-derived clone may result from asymmetric cell divisions that lead to different cell fate in a homogenous microenvironment. The aim of this study was to demonstrate the extent of cell variety in single-cell-derived clones and propose a different strategy in treating cancer by observed phenotypic heterogeneity in cellular types. Additionally, the role of metabolic enzyme and housekeeping gene, glyceraldehyde-3-phosphate dehydrogenase (GAPDH), in cellular phenotype was evaluated in two breast cancer cell lines. Materials and Methods: Two GAPDH-overexpressing breast cancer cell lines, MCF7-GAPDH-RFP (MCF7-RFP) and MDA-MB-231-GAPDH-RFP (MDA-RFP), were established. Microscopic recordings were made at 12- $h$ intervals in single-cell-derived clones of both cell lines up to 8-10 days. Crystal violet and Hoechst 33342 (Hoechst), as well as specific cell-type (epithelial and mesenchymal) antibodies, were used for cytochemical and immunohistochemical staining analyses, respectively, at 3, 6 and 9 days during cell growth. Results: Three types of clones with distinct morphologies were identified as holo-, mero- and paraclones. The rates of colony survival during cell growth were 8.3 and $41.7 \%$ in MCF7-RFP and MDA-RFP, respectively. Although no significant difference was found in the colony forming efficiency (CFE) of both MCF7 and MDA$M D$-231 wild-type cells, a markedly significant difference was seen in the CFE of MCF7-RFP and MDA-RFP cells $(p=0.001)$. Wild-type cell-derived holoclones of both cell lines showed drug resistance to doxorubicin (Dox). However, levels of proliferating cell nuclear antigen (PCNA) and vimentin (VIM) marginally decreased in Dox-treated clones. Furthermore, high level of intraclonal heterogeneity was found for CD44, CD140a, VIM, fibronectin (FN), focal adhesion
\end{abstract}

Correspondence to: Prof. Sang Ho Lee, Ph.D., Division of Life Sciences, Korea University, Seoul 02841, Republic of Korea. Tel: +82 232903423, Fax: +82 29279028, e-mail: sangho@korea.ac.kr

Key Words: Heterogeneity, GAPDH, clonal analysis, surface markers, MCF7, MDA-MB-231. kinase (FAK), paxillin (PXN) and vinculin (VCL) in MCF7 and MDA clones during different stages of clonal development. Expressions of CDI40a, FN, VIM and FAK were induced in GAPDH-red fluorescent protein (RFP)-tagged clones of both cell lines. Conclusion: The GAPDH-RFP recombinant protein played an important role in morphological heterogeneity detection in early stages of clonal development. Moreover, phenotypic heterogeneity in clones, caused by the cells expressing specific antigens, such as CD44, CDI40a, FN, VIM, FAK and VCL, can be the right target for therapeutic drugs.

In genetically homogenous cell populations, the phenotypic heterogeneity is frequently observed in in vitro cell cultures even in a controlled environment (1). The phenotypic changes also occur in a homogenous environment and may result from asymmetric segregation during cell division that leads to changes in gene expression patterns (2). Human breast cancer is one of the highly heterogeneous lesions having morphologically distinct subtypes with different molecular and biochemical signatures $(3,4)$. High level of heterogeneity in breast cancer cell phenotypes is most probably due to the dynamic plasticity of the breast cancer microenvironment $(5,6)$. The main reason behind this dynamic plasticity and heterogeneity is the presence of the small population of stem-like cells called breast cancer stem cells (7). Tumor-initiating cells expressing $\mathrm{CD} 44^{+} / \mathrm{CD} 24^{-/ \text {low }}$ and CD140a play a major role in breast cancer heterogeneity (8-10). In previous studies, it was reported that mesenchymal stem cells (MSCs) are responsible for the initiation of epithelial-mesenchymal transition (EMT) leading to cancer progression (10). Cytoskeleton re-organization and upregulation of mesenchymal markers during EMT, such as fibronectin (FN), vimentin (VIM) and N-cadherin, can also influence cellular phenotypes (11). In tumor cells, focal adhesion kinases (FAK) have a dual function both in promoting tumor cell adhesion and acting as a survival signal to inhibit apoptosis (12). FAK loss reduces the number and size of mammospheres and down-regulates the expression of surface markers in mammary cancer stem cells (13). However, the role of EMT and cell migration in breast cancer heterogeneity are not yet very clear. 
Glyceraldehyde-3-phosphate dehydrogenase (GAPDH) was initially identified as a glycolytic enzyme (14). GAPDH is also involved in cell death regulation, cell survival, tumor progression, DNA replication, endocytosis, exocytosis, carcinogenesis and cytoskeletal organization (15-17). It is known that GAPDH induces transcriptional activity of androgen receptor in prostate cancer cells (18). Additionally, its role in prevention of lung injury in a lipopolysaccharidetreated murine model was recently established (19). In breast cancer cells, GAPDH expression is connected with cell proliferation and aggressiveness of tumors (20). However, the role of GAPDH is not yet revealed in cancer cell heterogeneity.

This study was designed to trace the phenotypic and functional heterogeneity in single-cell-derived clones by focusing on developmental stages in human breast cancer cell lines. Phenotypic variations among clones of same or different cell types were evaluated by cell clonogenicity and drug resistance assays. Spatiotemporal expressions of cell surface and cytoskeletal markers were evaluated during different growth periods.

\section{Materials and Methods}

Cell lines and drugs. The MCF7 and MDA-MB-231 cell lines were obtained from the American Type Culture Collection (ATTC; Manassas, VA, USA). The MCF7-GAPDH-RFP (MCF7-RFP) and MDA-GAPDH-RFP (MDA-RFP) cell lines were established. Hoechst 33342 (Hoechst; Sigma-Aldrich, Seoul, South Korea), G418 (Sigma-Aldrich, Seoul, South Korea) and doxorubicin (Dox; Sigma-Aldrich) were dissolved in autoclaved distilled water to make $20 \mathrm{mg} / \mathrm{ml}, 50 \mathrm{mg} / \mathrm{ml}$ and $1 \mathrm{mM}$ stock solutions, respectively. All stock solutions were aliquoted and distributed in small volumes and stored at $-70^{\circ} \mathrm{C}$ until use.

Cell culture and growth experiments. The cells were maintained as an adherent monolayer culture in high glucose Dulbecco's modified Eagle's medium (DMEM; Gibco, Grand Island, NY, USA). The medium was supplemented with $10 \%(\mathrm{v} / \mathrm{v})$ heat-inactivated fetal bovine serum (FBS; Welgene, Daegu, South Korea), $100 \mu \mathrm{g} / \mathrm{ml}$ penicillin and streptomycin (Sigma), sodium pyruvate $(110 \mathrm{mg} / \mathrm{l})$ and sodium bicarbonate $(3.7 \mathrm{~g} / \mathrm{l})$. The cells were grown on culture dishes (SPL Life Sciences, Seoul, Korea) and incubated in $5 \% \mathrm{CO}_{2}$ in air at $37^{\circ} \mathrm{C}$. The medium was changed every $48 \mathrm{~h}$. The cells were regularly sub-cultured after $80 \%$ confluency by trypsinization and dispersion.

Plasmid construction, transfection and establishment of stable cell lines. The human glyceraldehyde 3-phosphate dehydrogenase (GAPDH; 1015 bp) was inserted into the commercial vector DsRedN1 (Takara Korea Biomedical Inc., Seoul, Korea) to construct the GAPDH-red fluorescent protein (RFP) plasmid. Standard procedure for cloning was used to make the target construct followed by confirmation and purification with PhoenIX ${ }^{\mathrm{TM}}$ Maxiprep kit (Qbiogene, Carlsbad, CA, USA), and $1 \mu \mathrm{g} / \mu \mathrm{l}$ stock was aliquoted in small volumes and stored at $-70^{\circ} \mathrm{C}$. Cells $\left(1 \times 10^{7}\right)$ were transfected with $0.5 \mu \mathrm{g}$ plasmid by using Neon ${ }^{\circledR}$ transfection system
(Invitrogen, Carlsbad, CA, USA) following electroporation. After $48 \mathrm{~h}$, transfected cells were treated with $200 \mu \mathrm{g} / \mathrm{ml} \mathrm{G} 418$ followed by selection with mouth pipette for high GAPDH-overexpressing cells to establish stable cell lines.

Time-lapse clonal development assay. Single cell suspensions of MCF7-RFP and MDA-RFP cells $\left(2 \times 10^{3}\right)$ were seeded in $60 \mathrm{~mm}$ media-containing dishes. Five hours later, cells were selected under Nikon fluorescence microscope (ECLIPS, TE-2000; Nikon, Tokyo, Japan) and encircled for colony development analyses. The positions of cultured cell dishes were fixed on the microscopic stage by marking specific matching points. Marked position on culture dishes and over the stage of microscope were correctly matched at every $12 \mathrm{~h}$ for image recording until 10 15 days. The standard criteria for clone size were based on total possible area observed by 200x lens under Nikon fluorescence microscope. The quantitative data for cell number per clone was collected from a various independent set of experiments.

Hoechst 33342 (Hoechst) analysis of developing clones. Single-cellderived clones were stained with $10 \mu \mathrm{g} / \mathrm{ml}$ Hoechst at $37^{\circ} \mathrm{C}$ in a $5 \%$ $\mathrm{CO}_{2}$ incubator for $20 \mathrm{~min}$ and washed three times with phosphatebuffered saline (PBS) followed by DMEM containing FBS. Hoechst-stained images of clones were recorded by using the camera of a fluorescence microscope (Nikon) connected with a FLEX (Diagnostic Instrument, Inc., Sterling Heights, MI, USA), using SPOT software ver. 4.6 (2006; Diagnostic Instrument, Inc.).

Clonogenic assay. Four different cell lines were seeded at a density of 200 cells per $35-\mathrm{mm}$ culture plates. Colonies were fixed with an ice-cold methanol-acetic acid solution $(3: 1)$ for $30 \mathrm{~min}$ at $-20^{\circ} \mathrm{C}$ at the end of the week and stained with $1 \%$ crystal violet for $30 \mathrm{~min}$ at room temperature. The images were recorded with a low magnification $(4 \times 0.13)$ lens in a bright field of a microscope with fixed digital camera (Nikon). The colony numbers per dish were counted and the mean number was obtained.

Multidrug resistance assay. Single-cell-derived holoclones were treated with Dox $(1.5 \mu \mathrm{g} / \mathrm{ml})$ for $24 \mathrm{~h}$ as reported (21). Control and treated clones were fixed as described and nuclei were stained with 4' 6-diamidino-2-phenylindole dihydrochloride (DAPI; $5 \mu \mathrm{g} / \mathrm{ml}$ ) for $20 \mathrm{~min}$ in dark. For DAPI detection, images were recorded under fluorescence lenses of a microscope connected to a digital camera.

Immunocytochemistry. Completely dissociated cells $\left(1 \sim 1.5 \times 10^{3}\right)$ were seeded on $0.1 \%$ gelatin-coated coverslips in $60-\mathrm{mm}$ culture dishes for 3, 6 days and 9 days and slandered immunocytochemistry method was used. Briefly, clones in different groups were washed with PBS three times and fixed with $4 \%$ paraformaldehyde for 20 min, followed by treatment with $0.26 \%$ ammonium chloride solution for $30 \mathrm{~min}$ at RT. HPEM buffer $(5 \mathrm{mM}$ HEPES, $5 \mathrm{mM}$ PIPES, $2 \mathrm{mM}$ EGTA, $1 \mathrm{mM} \mathrm{MgCl}$ ) with $0.5 \%$ Triton X-100 were used for extraction. The coverslips were incubated with antibodies to CD24, CD44, FAK, VCL, paxillin (PXN; dilution 1:100; Abcam, Cambridge, UK), PCNA, NF-kB, VIM, FN (dilution 1:150; Abcam), CD140a (dilution 1:50; Abcam) overnight at $4^{\circ} \mathrm{C}$. They were then washed with $0.05 \%$ Tween 20 containing PBS for $40 \mathrm{~min}$ followed by incubation with mouse, rabbit and rat FITC-conjugated secondary antibodies (dilution 1:200) for $1.5 \mathrm{~h}$ at room temperature. DAPI $(2 \mu \mathrm{g} / \mathrm{ml})$ was used as a nuclear localizing stain. 
Statistical analysis. The percentage of total colony number and types of different cell lines were analyzed by Student's $t$-test using SPSS ver. 12.0 (SPSS Inc. Chicago, IL, USA). The differences in colony number and colony subtype were considered statistically significant at $p \leq 0.05$.

\section{Results}

Phenotypic variability in colony morphologies in a singlecell-derived clonal population of breast cancer cells. The GAPDH-overexpressing cells were selected for clonal analysis compared to wild-type breast cancer cells. Both MCF7-RFP and MDA-RFP cell types have distinctive outlines with clear-looking nuclei (Figure 1A). MCF7-RFP cells formed colonies of 4 5 cells in 3 days followed by distinguishable morphologies after a week. Irregular shapes with a scattered pattern of cells were found in MDA-RFP clones at initial stages of colonies' development. MDA-RFP cells adopt to form colonies with proper shape after few cell divisions. High level of variations among cells size and shapes of clones were observed among clones with increasing number of cells. Based on morphological features, colonies were classified into holo-, mero- and paraclones (Figure 1B). MCF7-RFP holoclones were clusters of homogenous small and tightly packed cells with regular and smooth colony borderlines. In the case of MDA-RFP cells, the boundaries of holoclones were not very smooth. MCF7RFP meroclones showed loose cellular connection and had a limited proliferative capacity, while an irregular boundary line was observed in MDA-RFP clones. MCF7-RFP paraclones have dispersed and limited number of large size cells with undefined border. MDA-RFP paraclones showed few small size cells and scattered pattern. High rates of cell proliferation and survival were observed in holo- and meroclones compared to those of paraclones. A cell, having a mesenchymal-like phenotype, was observed to move toward the periphery of clones during clonal development. However, cells with epithelial phenotype were also found in the border area of the individual clone. Compared to MCF7RFP, MDA-RFP clonal cells showed higher proliferative potential and survival rate during clonal development. A total of $8.33 \%$ MCF7-RFP cells scored 100 cells per clone in 16 days, while $41.88 \%$ MDA-RFP cells achieved the same number in a week (Figure $1 \mathrm{C}$ and $\mathrm{D}$ ).

Single cells possess differential capacities of making colony subtypes in different cell lines. The types of colonies represent the proliferative potential and stem cell-like characteristics of a cell line. To evaluate the functional heterogeneity, clonogenic efficiency was compared among selected cell lines (Figure 2A). The percent colony-forming efficiency in monoculture conditions for MCF7, MDA-MB231, MCF7-RFP and MDA-RFP were 76.2, 77.0, 28.8 and $73.1 \%$, respectively (Figure $2 \mathrm{~B}$ ). The produced holo-, mero- and paraclones were 10.9, 31.8, 32.1 and 13.5, 45.0, 25.8\% in MCF7 and MDA-MD-231 cells, respectively. In GAPDHoverexpressing cells, the produced holo-, mero- and paraclones were $1.1,13.2,9.6$ and $9.9,50.4,24.4 \%$ in MCF7-RFP and MDA-RFP cells, respectively (Figure 2B).

Single-cell-derived holoclones have the potential of drugresistance to Dox. To assess whether single-cell-derived holoclones have stemness, we evaluated their responses to Dox. No significant differences were observed in colony morphologies in wild-type clones with Dox compared to control at $24 \mathrm{~h}$ (Figure 2C). Nuclear fragmentation in a fraction of cells' nuclei of wild-type clones was detected by DAPI staining (Figure 2C). Immunocytochemistry results showed that Dox treatment down-regulated the proliferating cell nuclear antigen (PCNA) expression in MCF7 holoclones. The nuclear factor (NF)-kB expression increased slightly, while vimentin (VIM) expression was similarly decreased in Dox-treated clones. Compared to MCF7, the expression pattern of PCNA, NF-kB and VIM was not significantly down-regulated in MDA-MB-231 clones (Figure 2D).

Cancer stem cell-related surface markers are differentially expressed in single-cell-derived clones. To understand the phenotypic heterogeneity, the spatiotemporal expressions of cancer stem cell(csc)-associated surface markers; i.e. CD24, CD144 and CD140a, were evaluated during clonal development. No expression was detected for CD24 in wildtype and GAPDH-overexpressing cell-derived clones except 6-day MDA-RFP clones. Positive expression of CD44 and CD140a was found in MCF7 and no expression in MDA wild-type clones. The ratios of CD44 (+) and CD140a (+) cells gradually increased in a time-dependent manner during the development of MCF7 wild-type colonies (Figure 3A). In GAPDH-overexpressing cells; i.e. MCF7-RFP, clones showed a strong expression of CD44 at day 3 only, while CD140a expression gradually increased in a time-dependent manner. In MDA-RFP clones, CD44 expression peaked at days 3 and 9, while CD140a expression gradually increased from day 3 to day 9 (Figure 3B).

EMT markers are differentially expressed in different types of single-cell-derived clones. To understand the specificity and complexity of breast tumors at the cellular level, we dissected the molecular signatures with single-cell analyses of breast cancer cells. Along with surface markers, the expression pattern of EMT markers; i.e. VIM and FN, was evaluated. VIM was strongly expressed in a differential manner in MCF7 and MDA-MB-231 clones at different stages of development. FN was expressed at day 3 and 6 in MCF7 clones, while, in MDA-MB-231 clones, differential expression of FN was found only at day 9 (Figure 4A). 

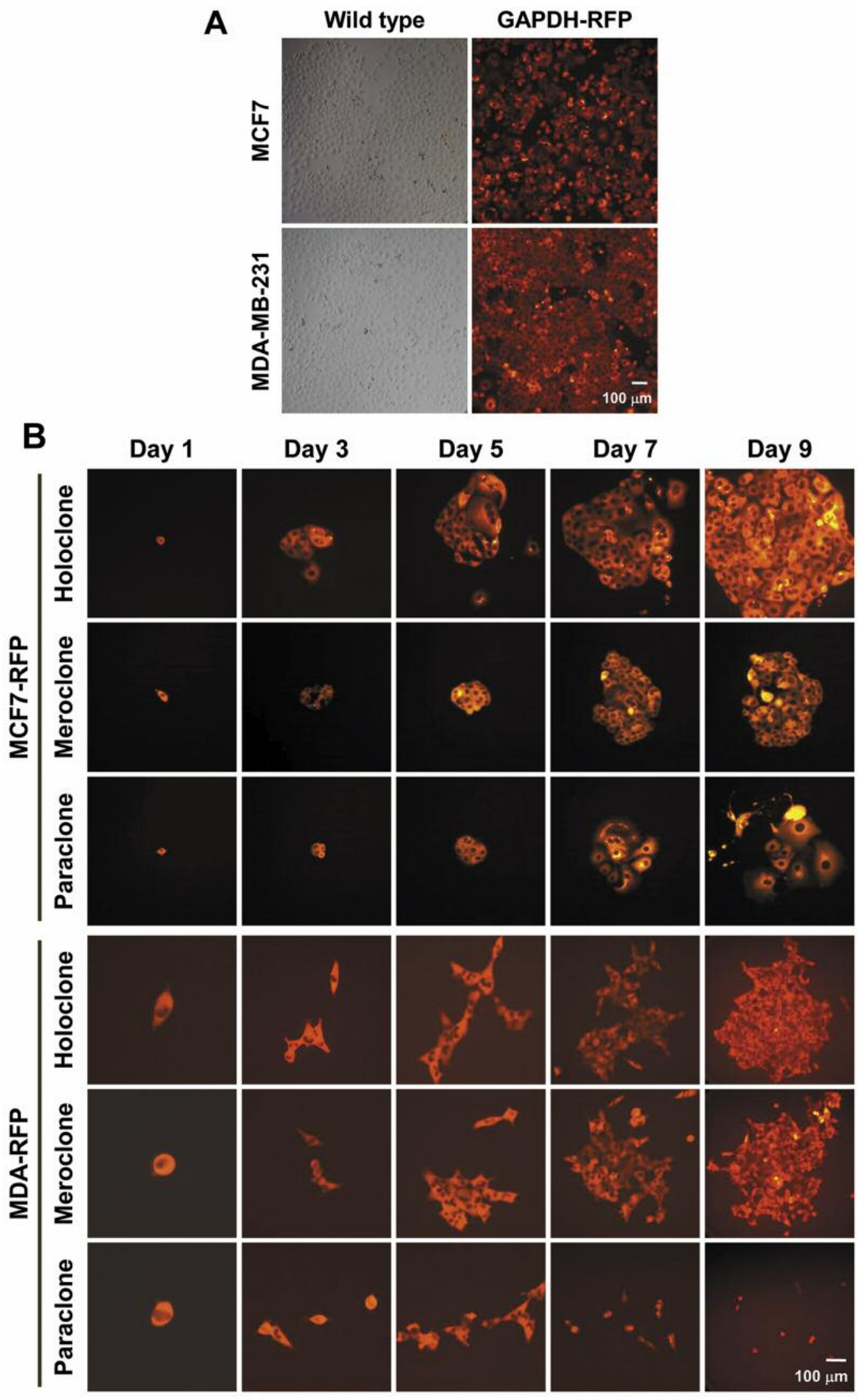

Figure 1. Continued 

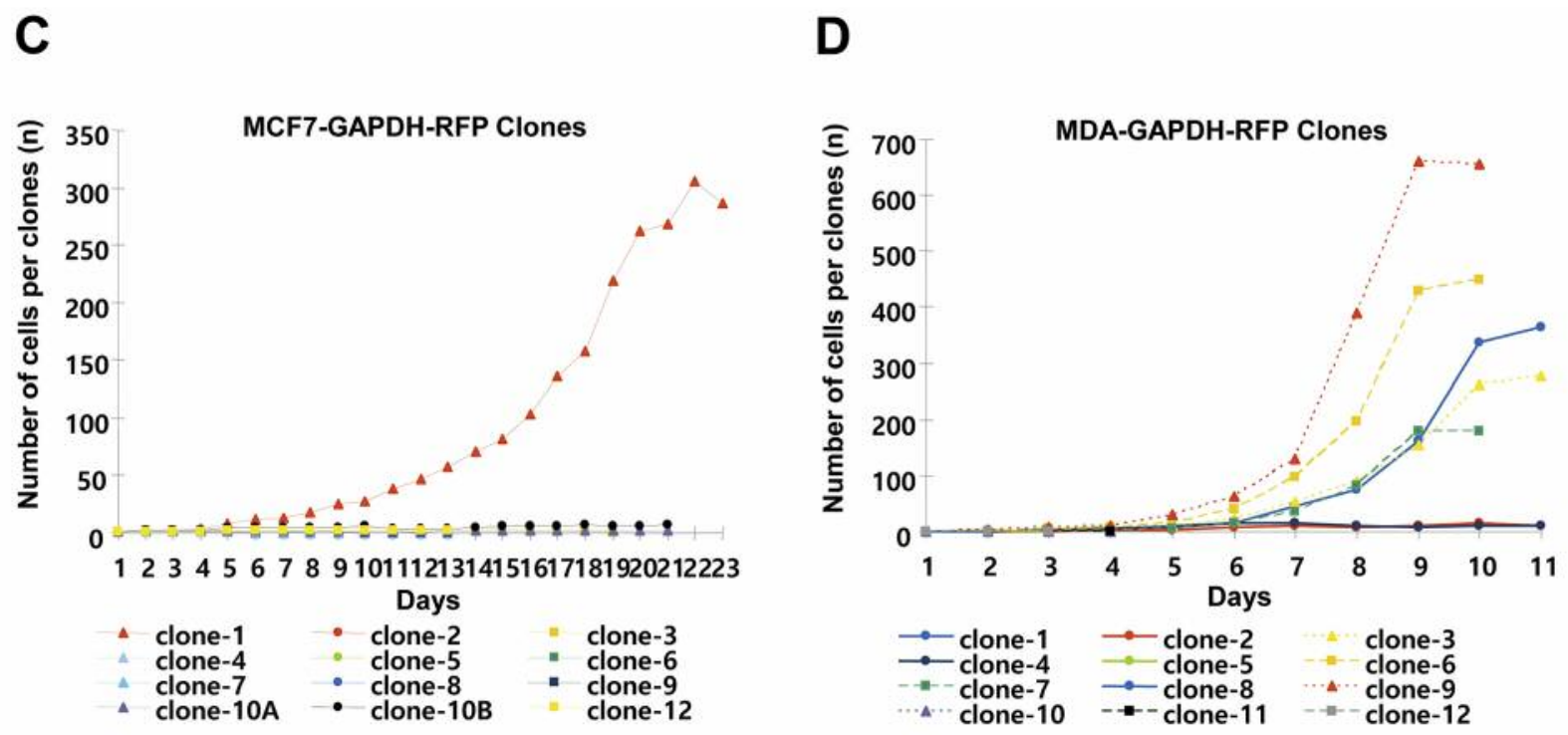

Figure 1. Analyses of developmental stages of single-cell-derived clones of breast cancer cell lines. A, Wild-type and GAPDH-overexpressing MCF7 and MDA-MB-231. B, Developmental stages of different types of clones of MCF7-RFP and MDA-RFP cell lines. C-D, Quantitative analysis of cell number per single-cell-derived clone of MCF7-RFP and MDA-RFP cells.

MCF7-RFP clones showed weak expression of VIM, while MDA-RFP clones strongly expressed VIM in different stages of development. MCF7-RFP clones strongly expressed FN, while differential expression was found in MDA-RFP clones (Figure 4B).

Spatiotemporal expression of focal adhesion molecules in single-cell-derived clones. To further characterize the migration and metastatic potentials of clonal cells, the expression patterns of FAK, PXN and VCL were evaluated. FAK was strongly expressed at days 3, 6 and, at the border of colonies, at 9 days. In MDA clones, FAK was differentially expressed in all stages of clonal development. PXN expression was found weak in all developmental stages of wild-type clones. VCL was gradually up-regulated in a time-dependent manner in MCF7 clones, while MDA-MB231 expressed VCL only in 9-day developed clones (Figure 5A). All MCF7-RFP and MDA-RFP clones showed strong differential expression of FAK during different stages of development. Compared to wild-type clones, PXN was upregulated in GAPDH-overexpressing clones. VCL expression gradually increased in MCF7-RFP clones. However, in MDA-RFP clones, strong differential expression of VCL was found only at day 6 (Figure 5B).

\section{Discussion}

The present study was conducted in order to trace the heterogeneity in single-cell-derived clones by using wild-type and GAPDH-overexpressing breast cancer MCF7 and MDA-
MB-231 cell lines. The major objective of this work was to establish an in vitro model for understanding the complexities of tumor development. Although the role of GAPDH in metabolism, apoptosis, cell survival and carcinogenicity has been profoundly studied, the impact of GAPDH in the heterogeneity of cancer cells has not yet been fully understood $(16,18)$. GAPDH-RFP recombinant proteins were overexpressed in two well-studied cell lines, MCF7 and MDA-MB-231, to uncover the mechanism of heterogeneity.

Due to cytoplasmic localization, GAPDH-RFP made it possible to trace morphological variations at individual cell level during clonal development. Based on cell morphology, epithelial and mesenchymal cells were identified. Their localization and mobility, however, were traced during different stages of growth. Tracking clonal growth every $12 \mathrm{~h}$ helped find out the pattern of cell division. At early growth stages, it was observed that either cell line followed symmetric or asymmetric cellular division. Previous studies have demonstrated that rates of symmetric and asymmetric cell division are responsible for the maintenance of CSCs (22). Cellular GAPDH-RFP overexpression was a very useful strategy to identify different morphologies. Unexpectedly, MCF7-RFP cells showed 5- and 2.5-fold lower cell survival and proliferation rates, respectively, compared to MDA-RFP. Clonal heterogeneity is characterized by differences in features, i.e. size, morphology, antigen expression and membrane composition, as well as behaviors, i.e. proliferation rate, cell-cell interaction, metastatic tendency and sensitivity to chemotherapy $(23,24)$. Also, the lowest efficiency of holoclone formation was found in MCF7-RFP cells. As 

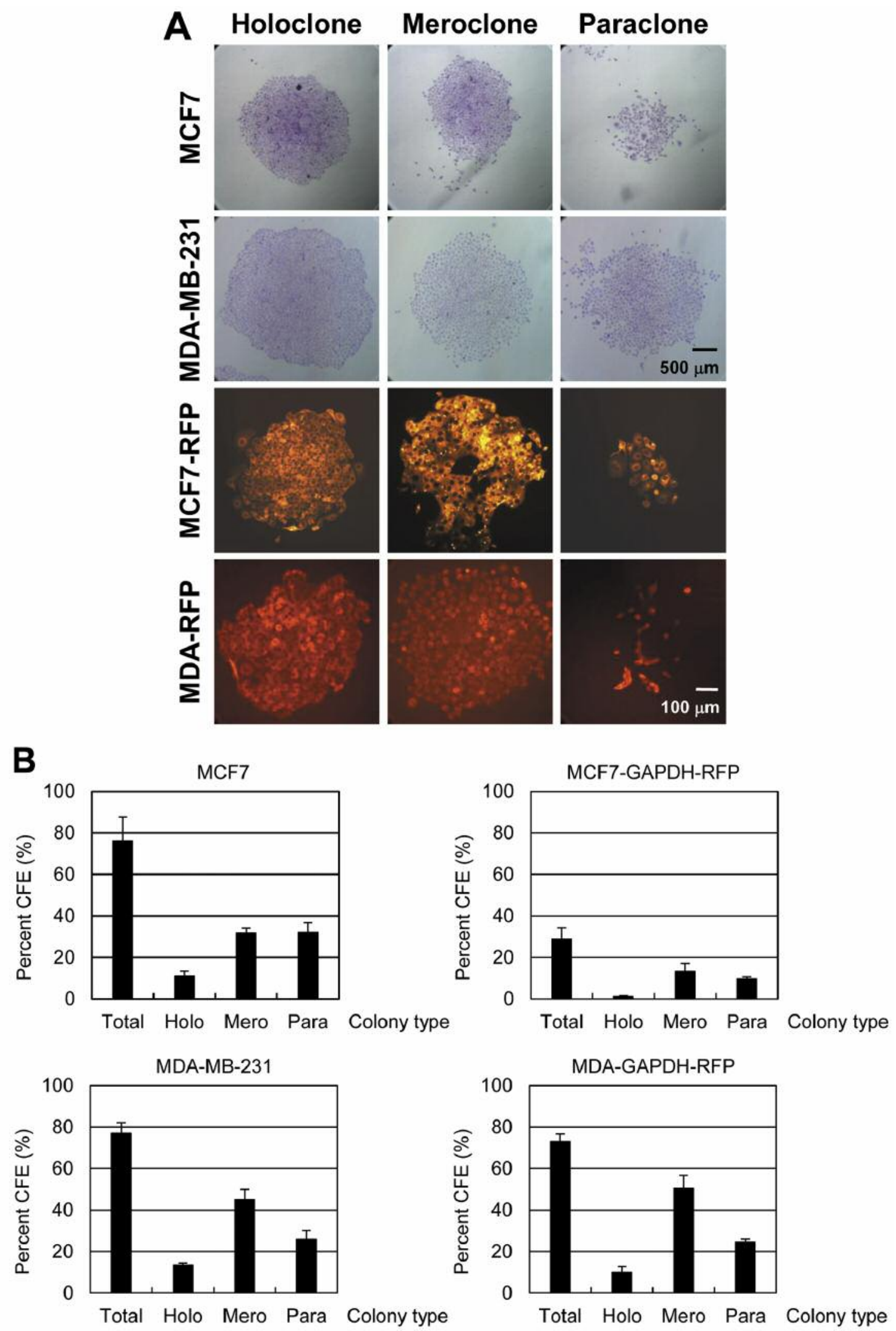

Figure 2. Continued 

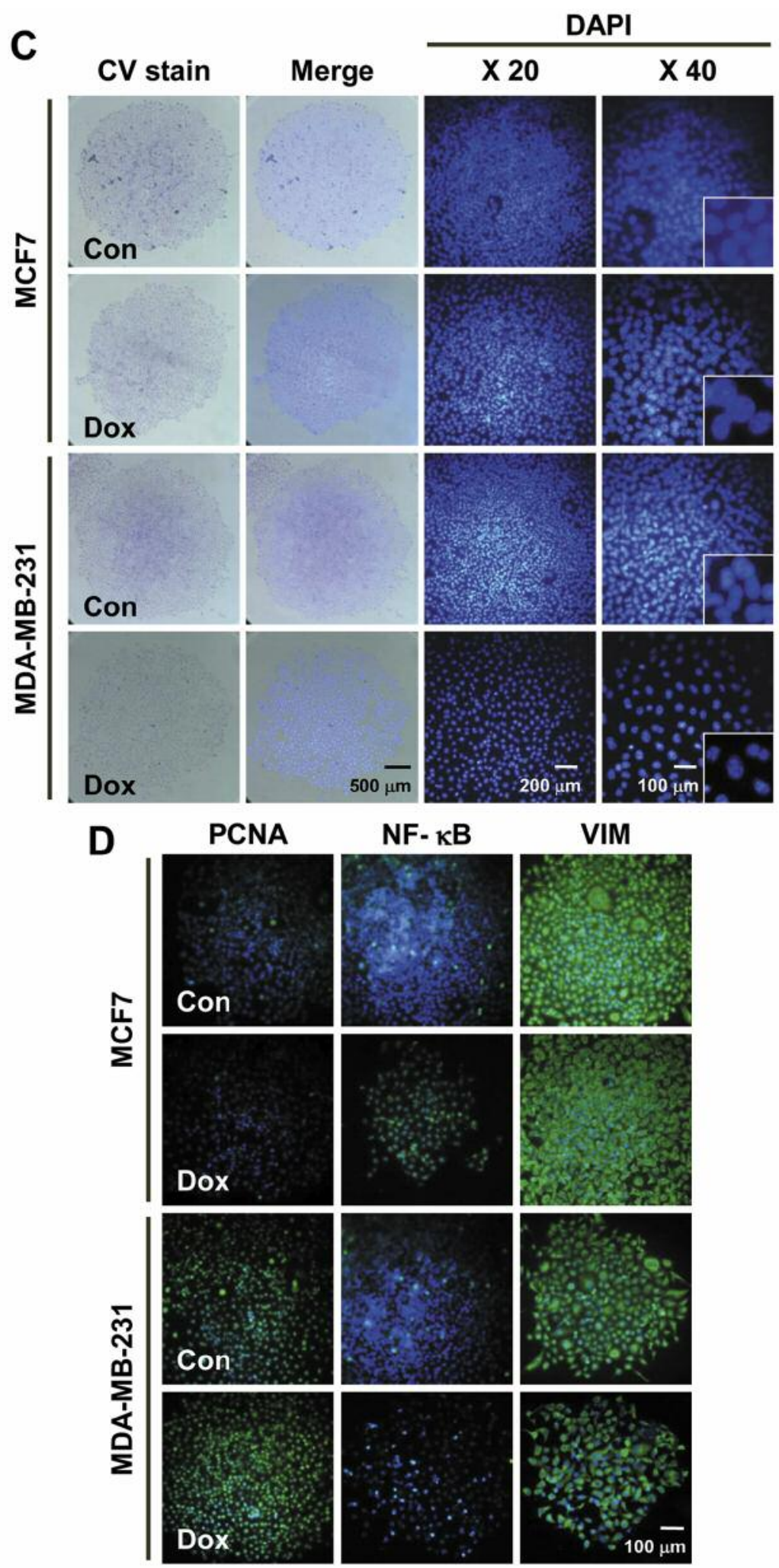

Figure 2. Morphological analyses of single-cell-derived clones of breast cancer cell lines. A, Colony types of wild-type and GAPDH-overexpressing MCF7 and MDA-MB-231 cells. B, Quantitative analysis of colony-forming efficiency (CFE) of different cell types. C, Crystal violet (CV) and DAPI staining for doxorubicin (Dox)-treated wild-type holoclones of MCF7 and MDA-MB-231 cells. D, Immunostaining of proliferating cell nuclear antigen (PCNA), nuclear factor (NF)-kB and vimentin (VIM) in Dox-treated MCF7 and MDA-MB-231 cells. 


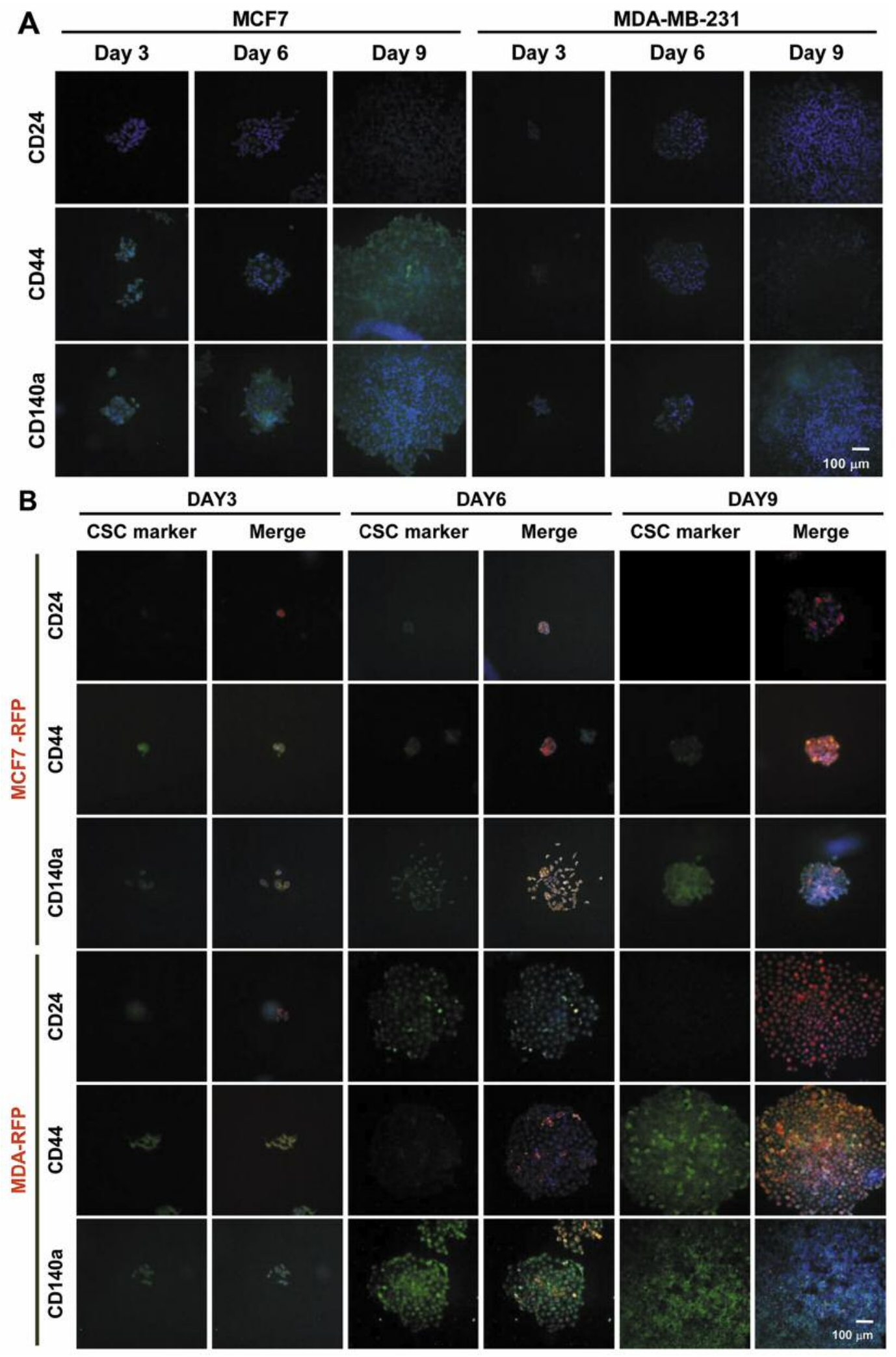

Figure 3. Immunostained cancer stem cell markers in single-cell-derived breast cancer clones. A, Expression of CD24, CD44 and CD140a in wildtype MCF7 and MDA-MB-231 cell-derived clones. B, Expression of CD24, CD44 and CD140a in GAPDH-overexpressing MCF7 and MDA-MB231 cell-derived clones. 
A
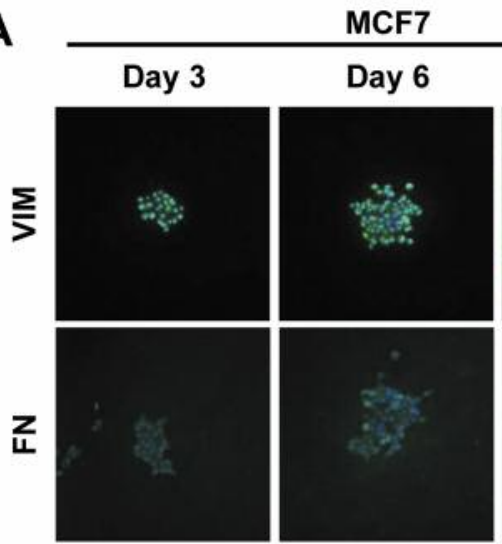

B

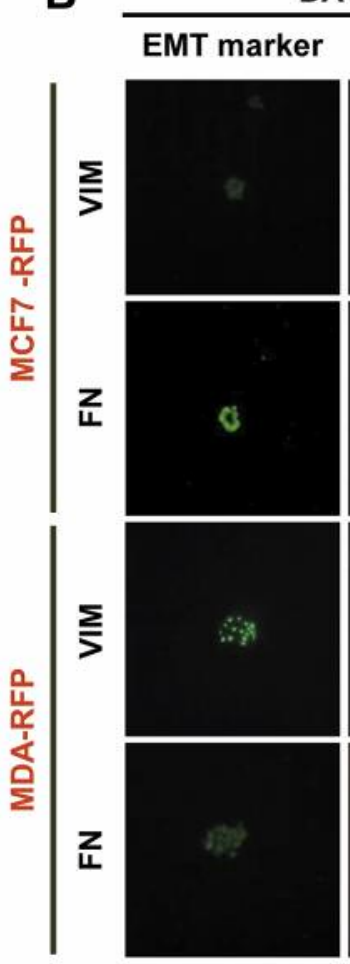

DAY3
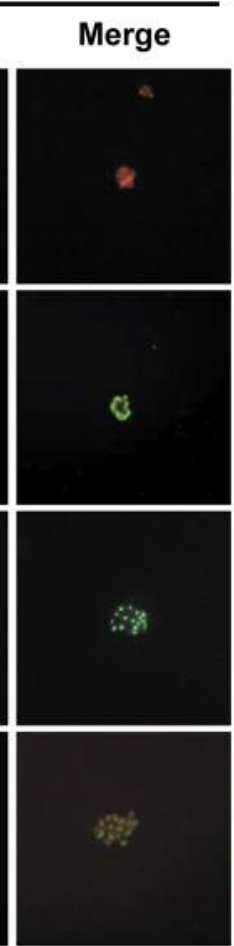

Day 9
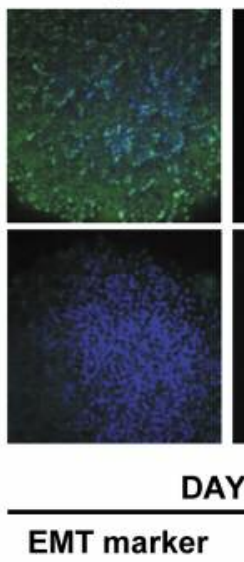
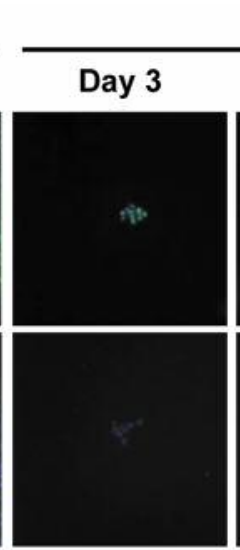

DAY6
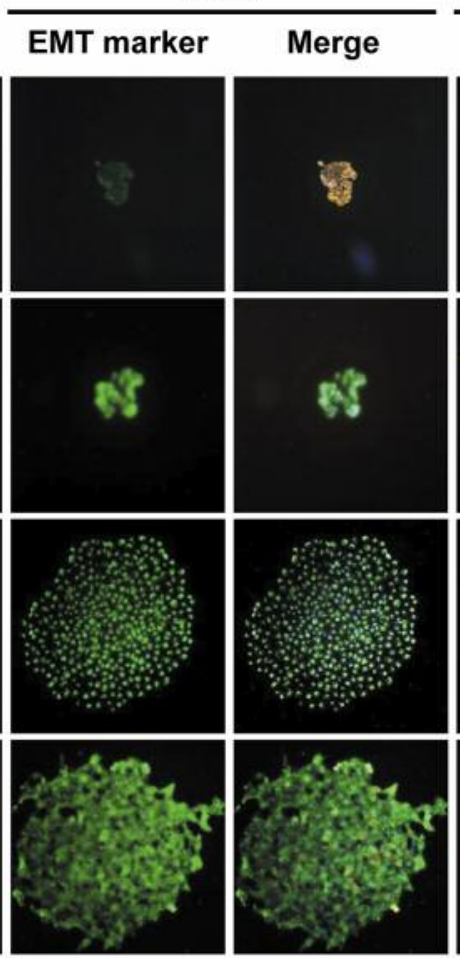

MDA-MB-231
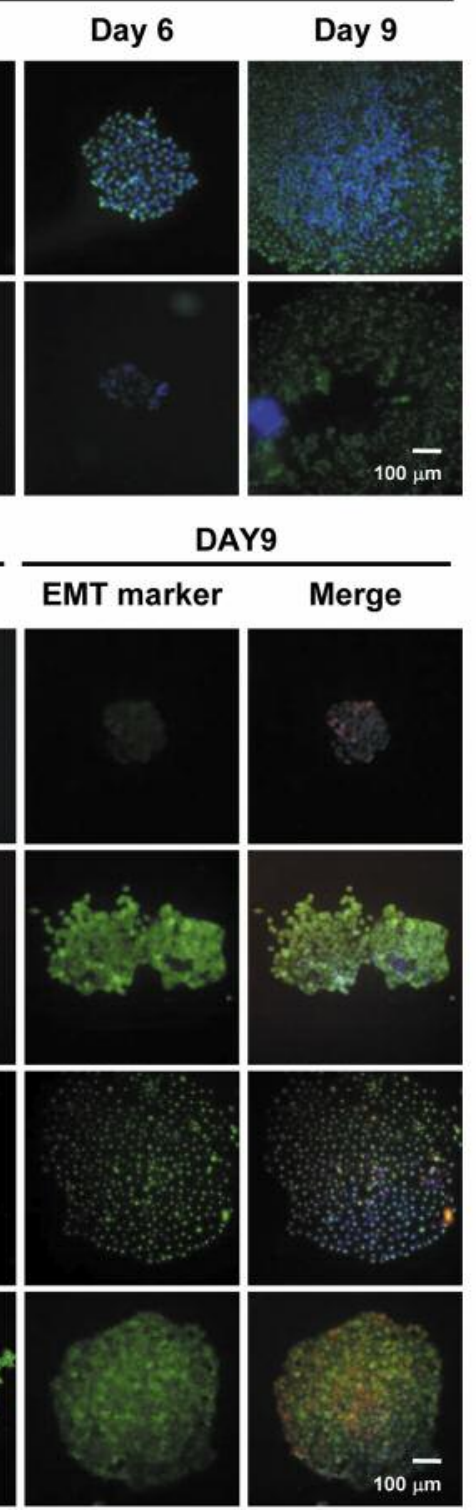

Figure 4. Immunostained EMT markers in single-cell-derived breast cancer clones. A, Expression of vimentin (VIM) and fibronectin (FN) in wildtype MCF7 and MDA-MB-231 cell-derived clones. B, Expression of VIM and FN in GAPDH-overexpressing MCF7 and MDA-MB-231 cell-derived clones.

reported, the percentage of holoclone formation of a cell line indicates the capacity of stemness (25). Our results show that GAPDH overexpression induced sensitivity in MCF7 cells compared to MDA-MB-231 cells.

The major characteristic of CSCs is to neutralize the effect of anticancer drugs via efflux transporter proteins $(21,26)$. In this study, the holoclones of MCF7 and MDA-MB-231 cells showed resistance to Dox. However, fragmented nuclei in a portion of cells of clones, as well as the marginal effect on expression levels of PCNA, NF-kB and VIM, were observed in MCF7 clones. Immunocytochemistry results of Dox-treated MDA-MB-231 clones showed that MDA-MB231 holoclones have more drug resistance capacities than MCF7 clones. As reported, Dox down-regulates PCNA, NF$\mathrm{kB}$ and VIM to control cell proliferation and EMT, respectively (27-30). Our results indicate that wild-type holoclones were not significantly affected by Dox and can be used as a model for CSC study of breast cancer. 


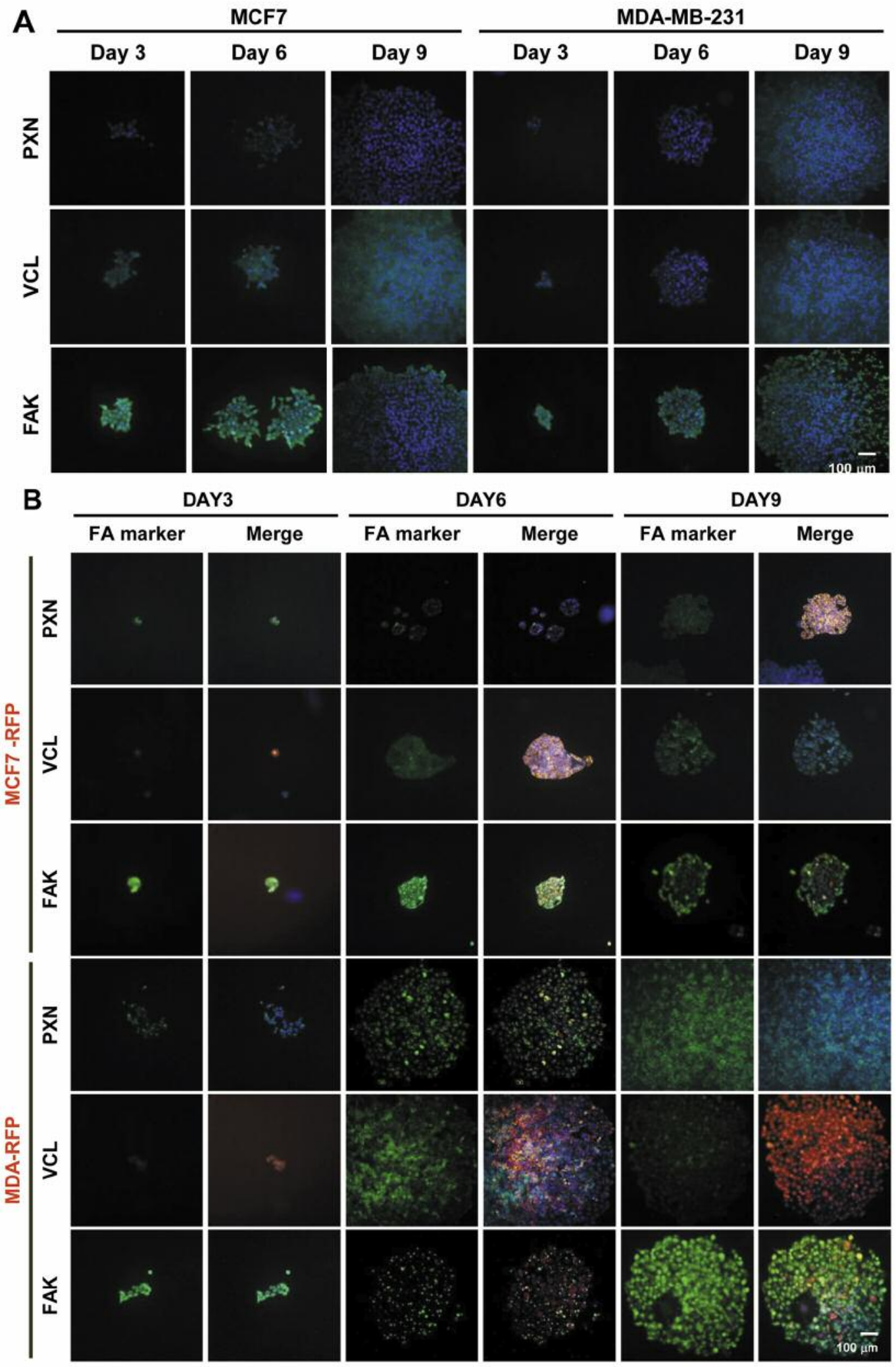

Figure 5. Expression of focal adhesion markers in single-cell-derived breast cancer clones. A, Expression of paxillin (PXN), vinculin (VCL) and focal adhesion kinase (FAK) in wild-type MCF7 and MDA-MB-231 cell-derived clones. B, Expression of PXN, VCL and FAK in GAPDHoverexpressing MCF7 and MDA-MB-231 cell-derived clones. FA, Focal expression. 
A small population of CSCs is being considered responsible for heterogeneity in different kinds of cancer. It appears that the asymmetric or symmetric cell division in CSCs depends on a possible interaction of key regulating factors. In the present study, variations were found in the expression pattern of CSCs markers, CD24, CD44 and CD140a, among same cell-derived, as well as clones of different cell lines. However, CD44 and CD140a expressions were weakly detected in MDA-MB-231 cell-derived clones. In a previous work, it has been reported that, compared to MCF7 cells, MDA-MB-231 cells exhibit more stemness (31). These results indicate that MDA-MB-231 clones possibly derive from CD44/CD140-/Low phenotype cells. Similar to the previous report, the differential expression of CD44 and CD140a further supports the hypothesis of heterogeneity in single-cell-derived breast cancer cell clones $(8,10,32,33)$. In GAPDH-overexpressing MCF7 and MDAMB-231 cells, the expression pattern showed the possible contribution of GAPDH in the up-regulation of CSC markers. Taken together, these results show that phenotypic heterogeneity exists among holoclones and GAPDH has an important role in their cellular phenotype determination.

Maintenance of the number of CSCs is strongly linked with the process of EMT, an epithelial switch to mesenchymal phenotype (34). Differential expression of VIM and FN in MCF7 and MDA-MB-231 cell-derived clones demonstrated the variations in cell phenotypes of wild-type clones. EMT, which involves loss of epithelial markers like E-cadherin and up-regulation of mesenchymal markers, such as FN and VIM, can also influence the cellular phenotype $(10,11)$. GAPDH overexpression down-regulated VIM in MCF7 cells, whereas FN was up-regulated in MCF7RFP and MDA-RFP cells. The expression pattern of EMT markers in wild-type clones was opposite to GAPDHoverexpressing clones. These results indicate that GAPDH affects cell phenotypes via influencing the expression of intermediate filaments and extracellular matrix proteins.

Cell motility and invasion depend on dynamic regulation of focal adhesions, peripheral actin structures and matrix metalloproteinase-mediated matrix degradation during migration (35). In this study, the high FAK-expressing cells exhibited mesenchymal phenotype and were found at the border of colonies of wild-type clones. Also, GAPDH affects FAK expression positively in GAPDH-overexpressing clones. Compared to wild-type clones, GAPDH-dependent up-regulation of PXN and VCL was also observed in GAPDH-overexpressing clones. Besides EMT, mobility and cell migration via integrin and FAK play an important role in metastasis $(36,37)$. In addition, the roles of FAK, PXN and VCL in cancer cell invasion and motility have already been studied $(35,38,39)$. However, the role of FAK, PXN and VCL is not clear in the heterogeneity of breast cancer cells. Our results show that heterogeneity in single-cell- derived clones is independent of origin. However, it mainly depends on an epithelial switch to mesenchymal phenotypes and vise versa.

In conclusion, we demonstrated the role of a single-cellderived clonal model to explore cellular heterogeneity in early stages of clonal development. This model can be used to investigate the initial stages of tumor development in vivo. Collectively, the presented data provided evidence on phenotypic and functional heterogeneity for same and/or different cell types at the single-cell level in breast cancer cells. This study will facilitate the exploration of new therapeutic strategies based on the single-cell-derived clonal analysis in in vitro systems.

\section{References}

1 Avery SV: Cell individuality: The bistability of competence development. Trends Microbiol 13(10): 459-462, 2005.

2 Singh SK, Hawkins C, Clarke ID, Squire JA, Bayani J, Hide T, Henkelman RM, Cusimano MD and Dirks PB: Identification of human brain tumour initiating cells. Nature 432(7015): 396-401, 2004.

3 Almendro V, Marusyk A and Polyak K: Cellular heterogeneity and molecular evolution in cancer. Annu Rev Pathol 8: 277-302, 2013.

4 Dawson SJ, Rueda OM, Aparicio S and Caldas C: A new genome-driven integrated classification of breast cancer and its implications. EMBO J 32(5): 617-628, 2013.

5 Place AE, Jin Huh S and Polyak K: The microenvironment in breast cancer progression: Biology and implications for treatment. Breast Cancer Res 13(6): 227, 2011.

6 Polyak K: Heterogeneity in breast cancer. J Clin Invest 121(10): 3786-3788, 2011.

7 Mannello F: Understanding breast cancer stem cell heterogeneity: Time to move on to a new research paradigm. BMC Med 11: 169, 2013.

8 Al-Hajj M, Wicha MS, Benito-Hernandez A, Morrison SJ and Clarke MF: Prospective identification of tumorigenic breast cancer cells. Proc Natl Acad Sci USA 100(7): 3983-3988, 2003.

9 Yu HG, Nam JO, Miller NL, Tanjoni I, Walsh C, Shi L, Kim L, Chen XL, Tomar A, Lim ST and Schlaepfer DD: p190RhoGEF (Rgnef) promotes colon carcinoma tumor progression via interaction with focal adhesion kinase. Cancer Res 71(2): 360-370, 2011.

10 Devarajan E, Song YH, Krishnappa S and Alt E: Epithelialmesenchymal transition in breast cancer lines is mediated through PDGF-D released by tissue-resident stem cells. Int J Cancer 131(5): 1023-1031, 2012.

11 Christiansen JJ and Rajasekaran AK: Reassessing epithelial to mesenchymal transition as a prerequisite for carcinoma invasion and metastasis. Cancer Res 66(17): 8319-8326, 2006.

12 Schlaepfer DD, Hanks SK, Hunter T and van der Geer P: Integrin-mediated signal transduction linked to Ras pathway by GRB2 binding to focal adhesion kinase. Nature 372(6508): 786$791,1994$.

13 Sulzmaier FJ, Jean C and Schlaepfer DD: FAK in cancer: Mechanistic findings and clinical applications. Nat Rev Cancer 14(9): 598-610, 2014. 
14 Zhang JY, Zhang F, Hong CQ, Giuliano AE, Cui XJ, Zhou GJ, Zhang GJ and Cui YK: Critical protein GAPDH and its regulatory mechanisms in cancer cells. Cancer Biol Med 12(1): 10-22, 2015.

15 Jung DW, Kim WH, Seo S, Oh E, Yim SH, Ha HH, Chang YT and Williams DR: Chemical targeting of GAPDH moonlighting function in cancer cells reveals its role in tubulin regulation. Chem Biol 21(11): 1533-1545, 2014.

16 Colell A, Green DR and Ricci JE: Novel roles for GAPDH in cell death and carcinogenesis. Cell Death Differ 16(12): 1573$1581,2009$.

17 Nicholls C, Li H and Liu JP: GAPDH: A common enzyme with uncommon functions. Clin Exp Pharmacol Physiol 39(8): 674679, 2012.

18 Harada N, Yasunaga R, Higashimura Y, Yamaji R, Fujimoto K, Moss J, Inui H and Nakano Y: Glyceraldehyde-3-phosphate dehydrogenase enhances transcriptional activity of androgen receptor in prostate cancer cells. J Biol Chem 282(31): 2265122661, 2007.

19 Takaoka Y, Goto S, Nakano T, Tseng HP, Yang SM, Kawamoto S, Ono K and Chen CL: Glyceraldehyde-3-phosphate dehydrogenase (GAPDH) prevents lipopolysaccharide (LPS)-induced, sepsisrelated severe acute lung injury in mice. Sci Rep 4: 5204, 2014.

20 Revillion F, Pawlowski V, Hornez L and Peyrat JP: Glyceraldehyde-3-phosphate dehydrogenase gene expression in human breast cancer. Eur J Cancer 36(8): 1038-1042, 2000.

21 Miloszewska J, Gos M, Przybyszewska M, Trembacz H, Koronkiewicz M and Janik P: Mouse sarcoma L1 cell line holoclones have a stemness signature. Cell Prolif 43(3): 229234, 2010.

22 Hwang WL, Jiang JK, Yang SH, Huang TS, Lan HY, Teng HW, Yang CY, Tsai YP, Lin CH, Wang HW and Yang MH: MicroRNA146a directs the symmetric division of Snail-dominant colorectal cancer stem cells. Nat Cell Biol 16(3): 268-280, 2014.

23 Campbell LL and Polyak K: Breast tumor heterogeneity: Cancer stem cells or clonal evolution? Cell Cycle 6(19): 2332-2338, 2007.

24 Durrett R, Foo J, Leder K, Mayberry J and Michor F: Intratumor heterogeneity in evolutionary models of tumor progression. Genetics 188(2): 461-477, 2011.

25 Beaver CM, Ahmed A and Masters JR: Clonogenicity: Holoclones and meroclones contain stem cells. PLoS One 9(2): e89834, 2014.

26 Vinogradov S and Wei X: Cancer stem cells and drug resistance: The potential of nanomedicine. Nanomedicine (Lond) 7(4): 597615, 2012

27 Qiao L, Pizzolo G and Melamed MR: Effects of selected chemotherapeutic agents on PCNA expression in prostate carcinoma cell lines. Urol Res 22(3): 171-176, 1994.

28 Ho WC, Dickson KM and Barker PA: Nuclear factor-kappaB induced by doxorubicin is deficient in phosphorylation and acetylation and represses nuclear factor-kappaB-dependent transcription in cancer cells. Cancer Res 65(10): 4273-4281, 2005.
29 Wang S, Kotamraju S, Konorev E, Kalivendi S, Joseph J and Kalyanaraman B: Activation of nuclear factor-kappaB during doxorubicin-induced apoptosis in endothelial cells and myocytes is pro-apoptotic: The role of hydrogen peroxide. Biochem $\mathrm{J}$ 367(Pt 3): 729-740, 2002.

30 Liu Y, Du FY, Chen W, Fu PF, Yao MY and Zheng SS: G15 sensitizes epithelial breast cancer cells to doxorubicin by preventing epithelial-mesenchymal transition through inhibition of GPR30. Am J Trans1 Res 7(5): 967-975, 2015.

31 Fillmore CM and Kuperwasser C: Human breast cancer cell lines contain stem-like cells that self-renew, give rise to phenotypically diverse progeny and survive chemotherapy. Breast Cancer Res 10(2): R25, 2008.

32 Kim Y, Kim E, Wu Q, Guryanova O, Hitomi M, Lathia JD, Serwanski D, Sloan AE, Weil RJ, Lee J, Nishiyama A, Bao S, Hjelmeland $\mathrm{AB}$ and Rich JN: Platelet-derived growth factor receptors differentially inform intertumoral and intratumoral heterogeneity. Genes Dev 26(11): 1247-1262, 2012.

33 Leth-Larsen R, Terp MG, Christensen AG, Elias D, Kuhlwein T, Jensen ON, Petersen OW and Ditzel HJ: Functional heterogeneity within the CD44 high human breast cancer stem cell-like compartment reveals a gene signature predictive of distant metastasis. Mol Med 18: 1109-1121, 2012.

34 Mani SA, Guo W, Liao MJ, Eaton EN, Ayyanan A, Zhou AY, Brooks M, Reinhard F, Zhang CC, Shipitsin M, Campbell LL, Polyak K, Brisken C, Yang J and Weinberg RA: The epithelialmesenchymal transition generates cells with properties of stem cells. Cell 133(4): 704-715, 2008.

35 Tomar A and Schlaepfer DD: Focal adhesion kinase: Switching between GAPs and GEFs in the regulation of cell motility. Curr Opin Cell Biol 21(5): 676-683, 2009.

36 Zhao X and Guan JL: Focal adhesion kinase and its signaling pathways in cell migration and angiogenesis. Adv Drug Deliv Rev 63(8): 610-615, 2011.

37 Cance WG, Harris JE, Iacocca MV, Roche E, Yang X, Chang J, Simkins $\mathrm{S}$ and $\mathrm{Xu} \mathrm{L}$ : Immunohistochemical analyses of focal adhesion kinase expression in benign and malignant human breast and colon tissues: Correlation with preinvasive and invasive phenotypes. Clin Cancer Res 6(6): 2417-2423, 2000.

38 Chen B, Xia L, Xu CS, Xiao F and Wang YF: Paxillin functions as an oncogene in human gliomas by promoting cell migration and invasion. Onco Targets Ther 9: 6935-6943, 2016.

39 Subauste MC, Pertz O, Adamson ED, Turner CE, Junger S and Hahn KM: Vinculin modulation of paxillin-FAK interactions regulates ERK to control survival and motility. J Cell Biol 165(3): 371-381, 2004. 